\title{
The Feasibility of Spinal Stereotactic Radiosurgery for Spinal Metastasis with Epidural Cord Compression
}

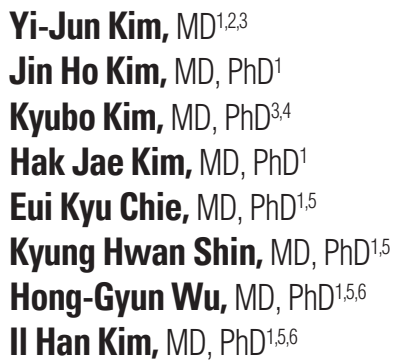

${ }^{1}$ Department of Radiation Oncology, Seoul National University College of Medicine, Seoul, ${ }^{2}$ Center for Precision Medicine, Seoul National University Hospital, Seoul, ${ }^{3}$ Department of Radiation Oncology, Graduate School of Medicine, Ewha Womans University, Seoul, ${ }^{4}$ Department of Radiation Oncology, Ewha Womans University College of Medicine, Seoul, ${ }^{5}$ Institute of Radiation Medicine, Seoul National University Medical Research Center, Seoul, ${ }^{6}$ Cancer Research Institute, Seoul National University, Seoul, Korea

Correspondence: Jin Ho Kim, MD, PhD Department of Radiation Oncology, Seoul National University College of Medicine, 103 Daehak-ro, Jongno-gu, Seoul 03080, Korea Tel: 82-2-2072-4767

Fax: 82-2-765-3317

E-mail: jinho.kim.md@gmail.com

Received November 27, 2018

Accepted January 28, 2019

Published Online January 29, 2019

\section{Purpose}

The purpose of this study was to investigate the effectiveness and safety of spinal stereotactic radiosurgery (SRS) in treating spinal metastasis with epidural spinal cord compression (ESCC).

\section{Materials and Methods}

During 2013-2016, 149 regions of spinal metastasis in 105 patients treated with singlefraction (12-24 Gy) spinal SRS were reviewed. Cord compression of Bilsky grade 2 (with visible cerebrospinal fluid [CSF]) or 3 (no visible CSF) was defined as ESCC. Local progression (LP) and vertebral compression fracture (VCF) rates after SRS were evaluated using multivariate competing-risk regression analysis.

\section{Results}

The 1-year cumulative incidences of LP for Bilsky grades 0 ( $n=80), 1(n=39), 2(n=21)$, and $3(n=9)$ were $3.0 \%, 8.4 \%, 0 \%$, and $24.9 \%$, respectively. Bilsky grade 2 ESCC did not significantly increase the LP rate (no LP for grade 2). The 1-year cumulative incidences of VCF for Bilsky grades $0,1,2$, and 3 were $6.6 \%, 5.2 \%, 17.1 \%$, and $12.1 \%$, respectively. ESCC may increase VCF risk (subhazard ratio [SHR] for grade 2, 5.368; $p=0.035$; SHR for grade 3, 2.215; $p=0.460$ ). Complete or partial pain response rates after SRS were 79\%, 78\%, 53\%, and $63 \%$ for Bilsky grades $0,1,2$, and 3 , respectively $(p=0.008)$. No neurotoxicity of grade $\geq 3$ was observed.

\section{Conclusion}

Spinal SRS for spinal metastasis with Bilsky grade 2 ESCC did not increase the LP rate, was not associated with severe neurotoxicity, and showed moderate VCF and pain response rates. Bilsky grade 3 had a high LP rate.

\section{Introduction}

Epidural spinal cord compression (ESCC) is observed in $5 \%-15 \%$ of patients who die of cancer [1]. Surgical decompression followed by radiotherapy (RT) is considered the treatment of choice as long as the surgery is an available option [2]. Conventional RT alone (e.g., $30 \mathrm{~Gy}$ in 10 fractions) seems to be less effective than surgery [2]. Therefore, RT

\section{Key words}

Spine, Neoplasm metastasis, Spinal cord compression, Fractures, Compression, Radiosurgery, Disease progression alone may be the second choice for patients who do not meet the criteria for surgery.

Meanwhile, spinal stereotactic radiosurgery (SRS) has been established as a treatment for patients with metastatic spinal tumors $[3,4]$. SRS is less invasive, simpler, and more convenient than open surgery.

In SRS for ECSS, all of the following should be considered: effective dose for local control, risk of vertebral compression fracture (VCF), and spinal cord dose constraints. Spinal SRS 
for patients with ESCC to control spinal metastasis with minimal VCF risk, and without cord injury, can be a challenge. SRS doses of $>20$ Gy to the tumor have been associated with high local control rates [5]. However, doses of $>20 \mathrm{~Gy}$ increase the risk of pathologic fracture [6]. Simultaneously, spinal cord dose constraints need to be satisfied. Quantitative Analysis of Normal Tissue Effects in the Clinic (QUANTEC) has demonstrated that a maximum cord dose of $13 \mathrm{~Gy}$ in a single fraction in spine radiosurgery appears to be associated with a $<1 \%$ risk of injury [7].

This study aimed to investigate the effectiveness and safety of SRS in treating spinal metastasis with ESCC and to analyze treatment outcomes according to the Bilsky criteria for ESCC.

\section{Materials and Methods}

\section{Study population}

The indications for SRS in our institution are follows: (1) patients with life expectancy $\geq 3$ months; (2) no definitive open surgery indications, such as neurologic symptoms that began within 48 hours, mechanical pain, or vertebral instability; (3) no conventional RT indications, such as a radiosensitive tumor, multiple metastases in more than three subsequent vertebrae, or a third irradiation at the same region; and (4) surgical indications with inoperable tumor/patient conditions.

In this study, patients who were treated with spinal SRS from January 2013 to December 2016 in a single institution were retrospectively reviewed. The inclusion criteria were as follows: (1) a pathologically confirmed malignant primary site, (2) no previous SRS at the treatment site, (3) no previous spine surgery at the SRS site, and (4) received a single-fraction SRS of $\geq 12$ Gy. Sacral metastasis cases were excluded due to absence of the thecal sac.

\section{SRS}

Linear accelerators (TrueBeam, Varian Medical Systems, Palo Alto, CA) or magnetic resonance imaging (MRI)-guided cobalt RT devices (ViewRay, ViewRay Technologies Inc., Oakwood Village, $\mathrm{OH}$ ) were used for the treatment. There were 145 cases that used the linear accelerator and two cases that used the MRI-guided cobalt RT device (all were Bilsky grade 0 ). When using the linear accelerator, a computed tomography (CT) simulation followed by MRI fusion of the spine was performed, and the fusion image was used for target delineation. When using the ViewRay device, the planning MRI was used for target delineation.
The International Spine Radiosurgery Consortium (ISRC) anatomic classification system was used to quantify the clinical target volume (CTV) [8]. ISRC 1 indicates the vertebral body. ISRC 2, 3, 4, 5, and 6 indicate the right pedicle, right lamina, spinous process, left lamina, and left pedicle, respectively. According to the ISRC consensus guidelines, the CTV includes the ISRC classification sites involving the gross tumor, as well as the adjacent normal marrow space. The planning target volume was made identical to the CTV or was generated by adding a 1-mm margin to the CTV.

The organs at risk were the spine, esophagus, and hypopharynx. The spine was delineated using MRI fusion, and the planning organ-at-risk volume was made by adding 1 $\mathrm{mm}$ to the spinal delineation. The dose constraint consisted of a maximum dose to the spinal cord of $14 \mathrm{~Gy}$, with $<10 \%$ of volume receiving $10 \mathrm{~Gy}$. The maximum dose to the cauda equina was $16 \mathrm{~Gy}$, with $<10 \%$ of volume receiving $12 \mathrm{~Gy}$. The maximum dose to a previously irradiated spinal cord was $13.5 \mathrm{~Gy}$; to the esophagus, $18 \mathrm{~Gy}$; to a previously irradiated esophagus, $17 \mathrm{~Gy}$; and to the hypopharynx, $19 \mathrm{~Gy}$.

The prescribed RT dose ranged from 12 to $24 \mathrm{~Gy}$. All patients were treated using volumetric modulated arc therapy. Patients were immobilized using vacuum cushions to the whole body and aquaplast splints for cervical spine treatment. Dexamethasone (2 $\mathrm{mg}$ three times a day for 3 days) was administered to the patients to prevent pain flares after SRS.

\section{ESCC classification}

Bilsky criteria were used to classify ESCC [9]. Bilsky grade 0 indicates bone-only disease, while grade 1 indicates a range from epidural impingement only (1-a) to deformation of the thecal sac with spinal cord abutment (1-c). Grade 2 indicates spinal cord compression with visible cerebrospinal fluid (CSF) around the cord, whereas grade 3 indicates no visible CSF around the cord.

\section{VCF risk classification}

VCF risk was classified using the Spinal Instability Neoplastic Score (SINS) [10]. Global spinal location of the tumor (junctional, mobile spine, semirigid, or rigid), pain, bone lesion quality (lytic, blastic, or mixed), spinal alignment, vertebral body collapse, and posterolateral involvement were scored, and cases were divided into three risk groups according to aggregated scores: stability (0-6), intermediate instability (7-12), and instability (13-18). 
Table 1. Patient characteristics according to Bilsky grade in spinal metastasis

\begin{tabular}{|c|c|c|c|c|c|c|}
\hline Characteristic & $\begin{array}{l}\text { No. of } \\
\text { regions }\end{array}$ & $\begin{array}{c}\text { Grade } 0 \\
(n=80)\end{array}$ & $\begin{array}{c}\text { Grade } 1 \\
(n=39)\end{array}$ & $\begin{array}{c}\text { Grade } 2 \\
(n=21)\end{array}$ & $\begin{array}{c}\text { Grade } 3 \\
(n=9)\end{array}$ & p-value ${ }^{a}$ \\
\hline \multicolumn{7}{|l|}{ Age (yr) } \\
\hline$\leq 55$ & 50 & $35(43.8)$ & $11(28.2)$ & $2(9.5)$ & $2(22.2)$ & 0.017 \\
\hline$>55$ & 99 & $45(56.3)$ & $28(71.8)$ & $19(90.5)$ & $7(77.8)$ & \\
\hline \multicolumn{7}{|l|}{ Sex } \\
\hline Male & 90 & $49(61.3)$ & $22(56.4)$ & $12(57.1)$ & $7(77.8)$ & 0.679 \\
\hline Female & 59 & $31(38.8)$ & $17(43.6)$ & $9(42.9)$ & $2(22.2)$ & \\
\hline \multicolumn{7}{|l|}{ ECOG performance status } \\
\hline $0-1$ & 137 & $77(96.3)$ & $34(87.2)$ & $19(90.5)$ & $7(77.8)$ & 0.127 \\
\hline $2-4$ & 12 & $3(3.8)$ & $5(12.8)$ & $2(9.5)$ & $2(22.2)$ & \\
\hline \multicolumn{7}{|l|}{ Primary tumor } \\
\hline Lung & 32 & $17(21.3)$ & $10(25.6)$ & $3(14.3)$ & $2(22.2)$ & 0.810 \\
\hline Breast & 25 & $14(17.5)$ & $7(17.9)$ & $4(19.0)$ & 0 & \\
\hline $\mathrm{HCC}$ & 33 & $17(21.3)$ & $8(20.5)$ & $5(23.8)$ & $3(33.3)$ & \\
\hline RCC & 10 & $4(5.0)$ & $3(7.7)$ & $1(4.8)$ & $2(22.2)$ & \\
\hline Others & 49 & $28(35.0)$ & $11(28.2)$ & $8(38.1)$ & $2(22.2)$ & \\
\hline \multicolumn{7}{|l|}{ Solitary metastasis } \\
\hline No & 129 & $68(85.0)$ & $34(87.2)$ & $19(90.5)$ & $8(88.9)$ & 0.919 \\
\hline Yes & 20 & $12(15.0)$ & $5(12.8)$ & $2(9.5)$ & $1(11.1)$ & \\
\hline \multicolumn{7}{|l|}{ SINS criteria } \\
\hline \multicolumn{7}{|l|}{ Tumor location } \\
\hline Junctional (occiput-C2, C7-T2, T11-L1, L5-S1) & 58 & $36(45.0)$ & $14(35.9)$ & $4(19.0)$ & $4(44.4)$ & 0.194 \\
\hline Mobile spine (C3-C6, L2-L4) & 44 & $25(31.3)$ & $10(25.6)$ & $6(28.6)$ & $3(33.3)$ & \\
\hline Semi-rigid (T3-T10) & 47 & $19(23.8)$ & $15(38.5)$ & $11(52.4)$ & $2(22.2)$ & \\
\hline \multicolumn{7}{|l|}{ Pain } \\
\hline Pain-free lesion & 38 & $24(30.0)$ & $7(17.9)$ & $6(28.6)$ & $1(11.1)$ & 0.012 \\
\hline Occasional pain but not mechanical & 78 & $41(51.3)$ & $27(69.2)$ & $5(23.8)$ & $5(55.6)$ & \\
\hline Mechanical & 33 & $15(18.8)$ & $5(12.8)$ & $10(47.6)$ & $3(33.3)$ & \\
\hline \multicolumn{7}{|l|}{ Bone lesion quality } \\
\hline Lytic & 102 & $59(73.8)$ & $26(66.7)$ & $11(52.4)$ & $6(66.7)$ & 0.008 \\
\hline Mixed (lytic/blastic) & 30 & $9(11.3)$ & $8(20.5)$ & $10(47.6)$ & $3(33.3)$ & \\
\hline Blastic & 17 & $12(15.0)$ & $5(12.8)$ & 0 & 0 & \\
\hline \multicolumn{7}{|l|}{ Radiographic spinal alignment } \\
\hline Normal alignment & 143 & $78(97.5)$ & $37(94.9)$ & $19(90.5)$ & $9(100)$ & 0.306 \\
\hline Kyphosis/Scoliosis & 5 & $2(2.5)$ & $2(5.1)$ & $1(4.8)$ & 0 & \\
\hline Subluxation/Translation & 1 & 0 & 0 & $1(4.8)$ & 0 & \\
\hline \multicolumn{7}{|l|}{ Vertebral body fracture } \\
\hline None of the below & 85 & $52(65.0)$ & $17(43.6)$ & $12(57.1)$ & $4(44.4)$ & 0.213 \\
\hline No collapse with $>50 \%$ body involved & 28 & $15(18.8)$ & $9(23.1)$ & $3(14.3)$ & $1(11.1)$ & \\
\hline$<50 \%$ collapse & 28 & $11(13.8)$ & $9(23.1)$ & $4(19.0)$ & $4(44.4)$ & \\
\hline$>50 \%$ collapse & 8 & $2(2.5)$ & $4(10.3)$ & $2(9.5)$ & 0 & \\
\hline \multicolumn{7}{|l|}{ Posterolateral involvement of spinal elements } \\
\hline None of the above & 83 & $56(70.0)$ & $20(51.3)$ & $5(23.8)$ & $2(22.2)$ & $<0.001$ \\
\hline Unilateral & 44 & $18(22.5)$ & $11(28.2)$ & $12(57.1)$ & $3(33.3)$ & \\
\hline Bilateral & 22 & $6(7.5)$ & $8(20.5)$ & $4(19.0)$ & $4(44.4)$ & \\
\hline \multicolumn{7}{|l|}{ SINS classification } \\
\hline Stability & 83 & $49(61.3)$ & $22(56.4)$ & $9(42.9)$ & $3(33.3)$ & 0.104 \\
\hline Intermediate stability & 62 & $30(37.5)$ & $17(43.6)$ & $10(47.6)$ & $5(55.6)$ & \\
\hline Instability & 4 & $1(1.3)$ & 0 & $2(9.5)$ & $1(11.1)$ & \\
\hline
\end{tabular}

(Continued to the next page) 
Table 1. Continued

\begin{tabular}{|c|c|c|c|c|c|c|}
\hline Characteristic & $\begin{array}{l}\text { No. of } \\
\text { regions }\end{array}$ & $\begin{array}{c}\text { Grade } 0 \\
(n=80)\end{array}$ & $\begin{array}{c}\text { Grade } 1 \\
(n=39)\end{array}$ & $\begin{array}{c}\text { Grade } 2 \\
(n=21)\end{array}$ & $\begin{array}{c}\text { Grade } 3 \\
(n=9)\end{array}$ & p-value ${ }^{a)}$ \\
\hline \multicolumn{7}{|l|}{ SRS dose (Gy) } \\
\hline$\leq 20$ & 135 & $66(82.5)$ & $39(100)$ & $21(100)$ & $9(100)$ & 0.004 \\
\hline$>20$ & 14 & $14(17.5)$ & 0 & 0 & 0 & \\
\hline \multicolumn{7}{|l|}{ SRS dose (Gy) } \\
\hline Median (range) & 149 & $18(12-24)$ & $18(14-20)$ & $18(13-20)$ & $18(16-20)$ & $0.009^{\mathrm{b})}$ \\
\hline \multicolumn{7}{|c|}{ ISRC sites number of treatment field } \\
\hline $1-3$ & 62 & $45(56.3)$ & $7(17.9)$ & $8(38.1)$ & $2(22.2)$ & 0.001 \\
\hline 4-6 & 87 & $35(43.8)$ & $32(82.1)$ & $13(61.9)$ & $7(77.8)$ & \\
\hline
\end{tabular}

Values are presented as number (\%) unless otherwise indicated. ECOG, Eastern Cooperative Oncology Group; HCC, hepatocellular carcinoma; RCC, renal cell carcinoma; SINS, Spinal Instability Neoplastic Score; SRS, stereotactic radiosurgery;

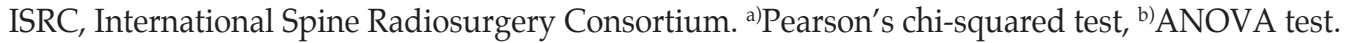

\section{Endpoints and follow-up}

The primary endpoints were local progression (LP) and VCF. The secondary endpoints were side effects and pain response rate.

LP-free survival was defined as the time from SRS to an occurrence of LP in the treated spine, patient death, or last follow-up. VCF progression-free survival was defined as the duration from SRS to an occurrence of a newly generated VCF or a VCF more progressed than a previous one, patient death, or last follow-up. LP and VCF were diagnosed based on serial changes in the radiologic images during the followup period, corresponding reports of radiologists, and clinical symptoms. Chart reviews were conducted three times by one physician (Y.J.K.), and equivocal cases were decided after discussion among two or more physicians.

Side effects were evaluated according to the Radiation Therapy Oncology Group-European Organisation for Research and Treatment of Cancer (RTOG-EORTC) criteria [11]. Criteria for acute toxicity covered day 1 (commencement of therapy) through day 90. Thereafter, criteria for late effects were utilized. Pain was scored using a visual analogue scale. Primary pain was scored on the day of the first visit. Pain after SRS was scored on every follow-up day. The minimum pain score after SRS was compared with the primary pain score before SRS.

The first visit was 1-2 weeks after SRS. Patients were followed up after 1 month and every 3 months thereafter. Spine MRI was also performed every 3 months or when patients expressed relevant symptoms. For analyses of LP and VCF, all radiologic images, such as spine, chest, and abdomen CT scans that were obtained for other reasons, were included in the assessment.

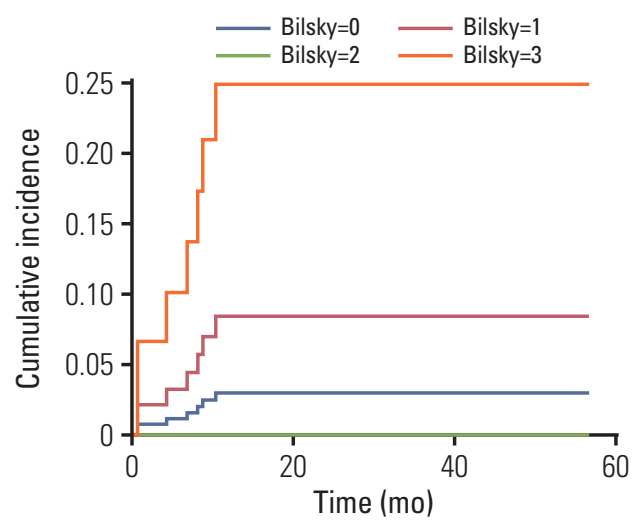

Fig. 1. Cumulative incidence function of local progression following univariate competing risk regression analysis in spinal metastasis treated with stereotactic radiosurgery.

\section{Statistical analysis}

Pearson's chi-squared test was used to compare characteristics and pain responses among groups categorized according to the Bilsky criteria. Death of a patient before LP or VCF was considered a competing risk. Cumulative incidences of LP and VCF rates were obtained using the univariate cumulative incidence function in the presence of competing risks, as suggested by Fine and Gray [12]. Subhazard ratios (SHR) of variables for LP and VCF were calculated using a univariate regression model.

Patient, tumor, and treatment variables that were significantly different among the Bilsky groups in the chi-square test, variables that had significant SHRs in the univariate regressions with $\mathrm{p}<0.1$, and variables that were considered clinically important were incorporated in the multivariate 
Table 2. Univariate and multivariate competing risk analyses of local progression after SRS in spinal metastasis

\begin{tabular}{|c|c|c|c|c|c|c|c|c|}
\hline \multirow{2}{*}{ Characteristic } & \multirow{2}{*}{$\begin{array}{l}\text { No. of } \\
\text { regions }\end{array}$} & \multirow{2}{*}{$\begin{array}{c}\text { No. of } \\
\text { events }(\%)\end{array}$} & \multicolumn{3}{|c|}{ Univariate } & \multicolumn{3}{|c|}{ Multivariate } \\
\hline & & & SHR & $95 \% \mathrm{CI}$ & $\overline{p-\text { value }^{\mathrm{a})}}$ & SHR & $95 \% \mathrm{CI}$ & $\overline{p-v a l u e^{b)}}$ \\
\hline \multicolumn{9}{|l|}{ Age (yr) } \\
\hline$\leq 55$ & 50 & $1(2)$ & \multicolumn{3}{|c|}{ Reference } & \multicolumn{3}{|c|}{ Reference } \\
\hline$>55$ & 99 & $6(6)$ & 3.409 & $0.430-27.026$ & 0.246 & 1.744 & $0.256-11.843$ & 0.569 \\
\hline \multicolumn{9}{|l|}{ Sex } \\
\hline Male & 90 & $6(7)$ & \multicolumn{3}{|c|}{ Reference } & & & \\
\hline Female & 59 & $1(2)$ & 0.257 & $0.0316-2.095$ & 0.204 & NA & & \\
\hline \multicolumn{9}{|l|}{ ECOG performance status } \\
\hline $0-1$ & 137 & $6(4)$ & \multicolumn{3}{|c|}{ Reference } & & & \\
\hline $2-4$ & 12 & $1(8)$ & 1.939 & $0.230-16.366$ & 0.543 & NA & & \\
\hline \multicolumn{9}{|l|}{ Primary tumor } \\
\hline Lung & 32 & $1(3)$ & \multicolumn{3}{|c|}{ Reference } & \multicolumn{3}{|c|}{ Reference } \\
\hline Breast & 25 & 0 & 0.000 & NA & $<0.001$ & 0.000 & NA & $<0.001$ \\
\hline $\mathrm{HCC}$ & 33 & $2(6)$ & 1.900 & $0.180-19.976$ & 0.594 & 3.526 & $0.388-32.061$ & 0.263 \\
\hline RCC & 10 & $2(20)$ & 7.885 & $0.783-79.411$ & 0.080 & 5.002 & $1.028-24.341$ & 0.046 \\
\hline Others & 49 & $2(4)$ & 1.362 & $0.130-14.333$ & 0.797 & 1.490 & $0.103-21.624$ & 0.770 \\
\hline \multicolumn{9}{|l|}{ Solitary metastasis } \\
\hline No & 129 & $7(5)$ & \multicolumn{3}{|c|}{ Reference } & \multicolumn{3}{|c|}{ Reference } \\
\hline Yes & 20 & 0 & 0.000 & NA & $<0.001$ & 0.000 & NA & $<0.001$ \\
\hline \multicolumn{9}{|l|}{ SINS criteria } \\
\hline \multicolumn{9}{|l|}{ Tumor location } \\
\hline $\begin{array}{l}\text { Junctional (occiput-C2, C7-T2, } \\
\text { T11-L1, L5-S1) }\end{array}$ & 58 & $4(7)$ & & Reference & & & & \\
\hline Mobile spine (C3-C6, L2-L4) & 44 & $1(2)$ & 0.338 & $0.038-3.004$ & 0.331 & NA & & \\
\hline Semi-rigid (T3-T10) & 47 & $2(4)$ & 0.649 & $0.121-3.496$ & 0.615 & & & \\
\hline \multicolumn{9}{|l|}{ Pain } \\
\hline Pain-free lesion & 38 & $2(5)$ & & Reference & & & & \\
\hline Occasional pain but not mechanical & 78 & $2(3)$ & 0.526 & $0.075-3.694$ & 0.518 & NA & & \\
\hline Mechanical & 33 & $3(9)$ & 1.978 & $0.337-11.620$ & 0.450 & & & \\
\hline \multicolumn{9}{|l|}{ Bone lesion quality } \\
\hline Lytic & 102 & $6(6)$ & & Reference & & & Reference & \\
\hline Mixed (lytic/blastic) & 30 & $1(3)$ & 0.596 & $0.072-4.962$ & 0.632 & 0.236 & $0.004-15.243$ & 0.497 \\
\hline Blastic & 17 & 0 & 0.000 & NA & $<0.001$ & 0.000 & NA & $<0.001$ \\
\hline Radiographic spinal alignment & & & & & & & & \\
\hline Normal alignment & 143 & $7(5)$ & & Reference & & & & \\
\hline Kyphosis/Scoliosis & 5 & 0 & 0.000 & NA & $<0.001$ & NA & & \\
\hline Subluxation/Translation & 1 & 0 & 0.000 & NA & $<0.001$ & & & \\
\hline Vertebral body fracture & & & & & & & & \\
\hline None of the below & 85 & $3(4)$ & & Reference & & & & \\
\hline No collapse with $>50 \%$ body involved & 28 & $1(4)$ & 1.045 & $0.107-10.186$ & 0.970 & NA & & \\
\hline$<50 \%$ collapse & 28 & $3(11)$ & 3.405 & $0.706-16.421$ & 0.127 & & & \\
\hline$>50 \%$ collapse & 8 & 0 & 0.000 & NA & $<0.001$ & & & \\
\hline $\begin{array}{l}\text { Posterolateral involvement of } \\
\text { spinal elements }\end{array}$ & & & & & & & & \\
\hline None of the above & 83 & $2(2)$ & & Reference & & & Reference & \\
\hline Unilateral & 44 & $3(7)$ & 3.178 & $0.546-18.488$ & 0.198 & 1.222 & $0.091-16.420$ & 0.880 \\
\hline Bilateral & 22 & $2(9)$ & 3.827 & $0.539-27.187$ & 0.180 & 1.576 & $0.208-11.909$ & 0.660 \\
\hline
\end{tabular}

(Continued to the next page) 
Table 2. Continued

\begin{tabular}{|c|c|c|c|c|c|c|c|c|}
\hline \multirow{2}{*}{ Characteristic } & \multirow{2}{*}{$\begin{array}{l}\text { No. of } \\
\text { regions }\end{array}$} & \multirow{2}{*}{$\begin{array}{c}\text { No. of } \\
\text { events }(\%)\end{array}$} & \multicolumn{3}{|c|}{ Univariate } & \multicolumn{3}{|c|}{ Multivariate } \\
\hline & & & SHR & $95 \% \mathrm{CI}$ & p-value $e^{a)}$ & SHR & $95 \% \mathrm{CI}$ & p-value ${ }^{\text {b) }}$ \\
\hline \multicolumn{9}{|l|}{ SINS classification } \\
\hline Stability & 83 & $3(4)$ & & Reference & & & & \\
\hline Intermediate stability & 62 & $3(5)$ & 1.436 & $0.293-7.043$ & 0.622 & NA & & \\
\hline Instability & 4 & $1(25)$ & 7.426 & $0.702-78.505$ & 0.096 & & & \\
\hline \multicolumn{9}{|l|}{ SRS dose (Gy) } \\
\hline$\leq 20$ & 135 & $7(5)$ & & Reference & & & Reference & \\
\hline$>20$ & 14 & 0 & 0.000 & NA & NA & 0.000 & NA & $<0.001$ \\
\hline \multicolumn{9}{|c|}{ ISRC sites number of treatment field } \\
\hline $1-3$ & 62 & 0 & & Reference & & & Reference & \\
\hline $4-6$ & 87 & $7(8)$ & NA & NA & NA & NA & NA & $<0.001$ \\
\hline \multicolumn{9}{|l|}{ Bilsky } \\
\hline Grade 0 & 80 & $2(3)$ & & Reference & & & Reference & \\
\hline Grade 1 & 39 & $3(8)$ & 2.917 & 0.494-17.214 & 0.237 & 1.160 & $0.078-17.203$ & 0.914 \\
\hline Grade 2 & 21 & 0 & 0.000 & NA & $<0.001$ & 0.000 & NA & $<0.001$ \\
\hline Grade 3 & 9 & $2(22)$ & 9.493 & $1.412-63.825$ & 0.021 & 4.521 & 0.353-57.852 & 0.246 \\
\hline
\end{tabular}

SRS, stereotactic radiosurgery; SHR, subhazard ratio; CI, confidence interval; NA, not applicable; ECOG, Eastern Cooperative Oncology Group; HCC, hepatocellular carcinoma; RCC, renal cell carcinoma; SINS, Spinal Instability Neoplastic Score; ISRC,

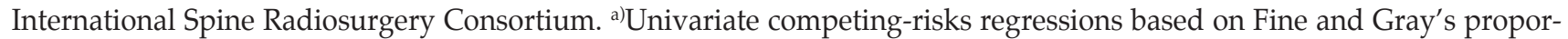
tional subhazards model, b) Multivariate competing-risks regressions based on Fine and Gray's proportional subhazards model.

competing-risks regression analysis.

All $\mathrm{p}$-values were two-sided, and $\mathrm{p}<0.05$ was considered statistically significant. The analyses were performed using Stata/MP 14.2 software (StataCorp., College Station, TX).

\section{Ethical statement}

This study was approved by an institutional review board at Seoul National University Hospital (no. 1705-049-853), and the requirement of informed consent for retrospective chart review was waived.

\section{Results}

\section{Patient characteristics}

The median follow-up period was 8.8 months (range, 0.5 to 56.6 months). There were 105 patients, 129 treatment cases, and 149 spinal regions (Table 1). ESCC (Bilsky grades 2-3) was correlated with more pain $(\mathrm{p}=0.012)$, non-blastic character $(\mathrm{p}=0.008)$, and posterolateral involvement $(\mathrm{p}<0.001)$. All patients in Bilsky grade 1-3 groups received doses of $\leq 20 \mathrm{~Gy}$

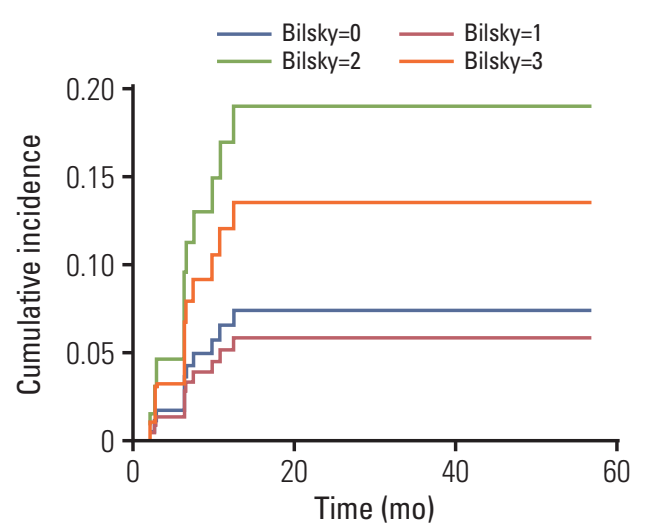

Fig. 2. Cumulative incidence function of vertebral compression fracture following univariate competing risk regression analysis in spinal metastasis treated with stereotactic radiosurgery.

$(p=0.004)$. The median dose was 18 Gy for all Bilsky groups.

\section{Local progression}

The median time of LP was 6.8 months (range, 0.6 to 10.3 months). During the follow-up period, only seven cases of 
Table 3. Univariate and multivariate competing risk analyses of vertebral compression fracture after SRS in spinal metastasis

\begin{tabular}{|c|c|c|c|c|c|c|c|c|}
\hline \multirow{2}{*}{ Characteristic } & \multirow{2}{*}{$\begin{array}{l}\text { No. of } \\
\text { regions }\end{array}$} & \multirow{2}{*}{$\begin{array}{c}\text { No. of } \\
\text { events }(\%)\end{array}$} & \multicolumn{3}{|c|}{ Univariate $^{\mathrm{a})}$} & \multicolumn{3}{|c|}{ Multivariate $^{\mathrm{b})}$} \\
\hline & & & SHR & $95 \% \mathrm{CI}$ & p-value & SHR & $95 \% \mathrm{CI}$ & p-value \\
\hline \multicolumn{9}{|l|}{ Age (yr) } \\
\hline$\leq 55$ & 50 & $2(4)$ & \multicolumn{3}{|c|}{ Reference } & \multicolumn{3}{|c|}{ Reference } \\
\hline$>55$ & 99 & $9(9)$ & 2.616 & $0.586-11.684$ & 0.208 & 1.595 & $0.268-9.479$ & 0.608 \\
\hline \multicolumn{9}{|l|}{ Sex } \\
\hline Male & 90 & $9(10)$ & \multicolumn{3}{|c|}{ Reference } & & & \\
\hline Female & 59 & $2(3)$ & 0.344 & $0.074-1.605$ & 0.175 & NA & & \\
\hline \multicolumn{9}{|l|}{ ECOG performance status } \\
\hline $0-1$ & 137 & $9(7)$ & \multicolumn{3}{|c|}{ Reference } & & & \\
\hline $2-4$ & 12 & $2(17)$ & 2.902 & $0.617-13.649$ & 0.177 & NA & & \\
\hline \multicolumn{9}{|l|}{ Primary tumor } \\
\hline Lung & 32 & $1(3)$ & \multicolumn{3}{|c|}{ Reference } & \multicolumn{3}{|c|}{ Reference } \\
\hline Breast & 25 & 0 & 0.000 & NA & $<0.001$ & 0.000 & NA & $<0.001$ \\
\hline $\mathrm{HCC}$ & 33 & $4(12)$ & 4.029 & $0.456-35.608$ & 0.210 & 4.369 & $0.372-51.299$ & 0.241 \\
\hline $\mathrm{RCC}$ & 10 & $1(10)$ & 3.619 & $0.233-56.192$ & 0.358 & 2.585 & $0.095-70.547$ & 0.573 \\
\hline Others & 49 & $5(10)$ & 3.548 & $0.427-29.471$ & 0.241 & 4.111 & $0.470-35.958$ & 0.201 \\
\hline \multicolumn{9}{|l|}{ Solitary metastasis } \\
\hline No & 129 & $10(8)$ & \multicolumn{3}{|c|}{ Reference } & & & \\
\hline Yes & 20 & $1(5)$ & 0.619 & $0.078-4.910$ & 0.649 & NA & & \\
\hline \multicolumn{9}{|l|}{ SINS criteria } \\
\hline \multicolumn{9}{|l|}{ Tumor location } \\
\hline $\begin{array}{l}\text { Junctional (occiput-C2, C7-T2, } \\
\text { T11-L1, L5-S1) }\end{array}$ & 58 & $5(9)$ & \multicolumn{3}{|c|}{ Reference } & & & \\
\hline Mobile spine (C3-C6, L2-L4) & 44 & $3(7)$ & 0.791 & $0.190-3.293$ & 0.747 & NA & & \\
\hline Semi-rigid (T3-T10) & 47 & $3(6)$ & 0.775 & $0.184-3.269$ & 0.728 & & & \\
\hline \multicolumn{9}{|l|}{ Pain } \\
\hline Pain-free lesion & 38 & $1(3)$ & \multicolumn{3}{|c|}{ Reference } & & Reference & \\
\hline Occasional pain but not mechanical & 78 & $7(9)$ & 3.829 & $0.475-30.813$ & 0.207 & 11.769 & $0.866-159.715$ & 0.064 \\
\hline Mechanical & 33 & $3(8)$ & 4.035 & $0.418-38.937$ & 0.228 & 5.370 & $0.518-55.718$ & 0.159 \\
\hline Bone lesion quality & & & & & & & & \\
\hline Lytic & 102 & $8(8)$ & & Reference & & & Reference & \\
\hline Mixed (lytic/blastic) & 30 & $2(7)$ & 0.923 & $0.191-4.464$ & 0.921 & 0.769 & $0.099-5.970$ & 0.801 \\
\hline Blastic & 17 & $1(6)$ & 0.615 & $0.084-4.493$ & 0.632 & 0.742 & 0.099-5.548 & 0.771 \\
\hline Radiographic spinal alignment & & & & & & & & \\
\hline Normal alignment & 143 & $10(7)$ & & Reference & & & Reference & \\
\hline Kyphosis/Scoliosis & 5 & $1(20)$ & 2.760 & $0.348-21.841$ & 0.336 & 8.749 & $1.069-71.643$ & 0.043 \\
\hline Subluxation/Translation & 1 & 0 & 0.000 & NA & $<0.001$ & 0.000 & NA & $<0.001$ \\
\hline Vertebral body fracture & & & & & & & & \\
\hline None of the below & 85 & $5(6)$ & & Reference & & & & \\
\hline No collapse with $>50 \%$ body involved & 28 & $2(7)$ & 1.224 & $0.240-6.247$ & 0.808 & NA & & \\
\hline$<50 \%$ collapse & 28 & $3(11)$ & 2.115 & $0.504-8.869$ & 0.306 & & & \\
\hline$>50 \%$ collapse & 8 & $1(13)$ & 2.107 & $0.272-16.221$ & 0.477 & & & \\
\hline $\begin{array}{l}\text { Posterolateral involvement of } \\
\text { spinal elements }\end{array}$ & & & & & & & & \\
\hline None of the above & 83 & $6(7)$ & & Reference & & & Reference & \\
\hline Unilateral & 44 & $4(9)$ & 1.393 & $0.397-4.887$ & 0.604 & 0.842 & 0.141-5.019 & 0.850 \\
\hline Bilateral & 22 & $1(5)$ & 0.616 & $0.074-5.157$ & 0.655 & 0.190 & $0.025-1.453$ & 0.109 \\
\hline
\end{tabular}

(Continued to the next page) 
Table 3. Continued

\begin{tabular}{|c|c|c|c|c|c|c|c|c|}
\hline \multirow{2}{*}{ Characteristic } & \multirow{2}{*}{$\begin{array}{l}\text { No. of } \\
\text { regions }\end{array}$} & \multirow{2}{*}{$\begin{array}{c}\text { No. of } \\
\text { events }(\%)\end{array}$} & \multicolumn{3}{|c|}{ Univariate $^{a)}$} & \multicolumn{3}{|c|}{ Multivariate $^{\mathrm{b})}$} \\
\hline & & & SHR & $95 \% \mathrm{CI}$ & p-value & SHR & $95 \% \mathrm{CI}$ & p-value \\
\hline \multicolumn{9}{|l|}{ SINS classification } \\
\hline Stability & 83 & $6(7)$ & & Reference & & & & \\
\hline Intermediate stability & 62 & $4(6)$ & 0.964 & $0.275-3.371$ & 0.954 & NA & & \\
\hline Instability & 4 & $1(25)$ & 3.491 & $0.414-29.456$ & 0.251 & & & \\
\hline \multicolumn{9}{|l|}{ SRS dose (Gy) } \\
\hline$\leq 20$ & 135 & $10(7)$ & & Reference & & & Reference & \\
\hline$>20$ & 14 & $1(7)$ & 1.065 & $0.136-9.332$ & 0.952 & 1.934 & $0.172-21.749$ & 0.593 \\
\hline \multicolumn{9}{|c|}{ ISRC sites number of treatment field } \\
\hline $1-3$ & 62 & $5(8)$ & & Reference & & & Reference & \\
\hline $4-6$ & 87 & $6(7)$ & 0.838 & $0.256-2.739$ & 0.770 & 0.807 & $0.098-6.628$ & 0.807 \\
\hline \multicolumn{9}{|l|}{ Bilsky } \\
\hline Grade 0 & 80 & $5(6)$ & & Reference & & & Reference & \\
\hline Grade 1 & 39 & $2(5)$ & 0.781 & $0.154-3.962$ & 0.766 & 0.753 & $0.150-3.780$ & 0.730 \\
\hline Grade 2 & 21 & $3(14)$ & 2.733 & $0.635-11.767$ & 0.177 & 5.368 & $1.129-25.530$ & 0.035 \\
\hline Grade 3 & 9 & $1(11)$ & 1.890 & $0.224-15.934$ & 0.559 & 2.215 & $0.269-18.248$ & 0.460 \\
\hline
\end{tabular}

SRS, stereotactic radiosurgery; SHR, subhazard ratio; CI, confidence interval; NA, not applicable; ECOG, Eastern Cooperative Oncology Group; HCC, hepatocellular carcinoma; RCC, renal cell carcinoma; SINS, Spinal Instability Neoplastic Score; ISRC,

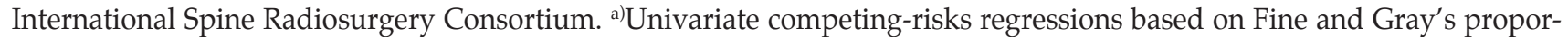
tional subhazards model, b) Multivariate competing-risks regressions based on Fine and Gray's proportional subhazards model.

LP were observed. Among them, there were two patients with Bilsky grade 0; 3 with Bilsky grade 1; 0 with Bilsky grade 2; and 2 with Bilsky grade 3. The cumulative incidence of LP at 1 year after SRS was $4.8 \%$. The 1-year cumulative incidence of LP for Bilsky grade 0 was 3.0\%; for Bilsky grade 1 , it was $8.4 \%$; for Bilsky grade 2, it was $0 \%$; and for Bilsky grade 3 , it was $24.9 \%$ (Fig. 1). On univariate competing-risk regression, Bilsky grade 3 was positively correlated with LP (compared with Bilsky grade 0; SHR for Bilsky grade 1, 2.917; 95\% confidence interval [CI], 0.494 to $17.214 ; \mathrm{p}=0.237$; SHR for Bilsky grade 2, not applicable due to no LP event; SHR for Bilsky grade 3, 9.493; 95\% CI, 1.412 to $63.825, \mathrm{p}=0.021$ ) (Table 2).

Patients whose primary lesions were breast lesions, who had a spinal lesion that was a solitary metastasis, whose lesions were blastic, who received a dose of $>20 \mathrm{~Gy}$, and whose treatment field was comprised of three or fewer ISRC sites, did not experience an LP.

On multivariate analysis of LP, compared with the Bilsky grade 0 group, the grades $1-3$ groups did not show statistically significant differences in LP (SHR for grade 1, 1.160; 95\% CI, 0.078 to 17.203; $\mathrm{p}=0.914$; no LP for grade 2; SHR for grade $3,4.521 ; 95 \% \mathrm{CI}, 0.353$ to $57.852 ; \mathrm{p}=0.246)$. Meanwhile, the following were statistically significant favorable factors: primary breast cancer, solitary metastasis, blastic lesion, RT dose of $>20 \mathrm{~Gy}$, and three or fewer ISRC sites in the treatment field ( $p<0.001$ for all). Primary renal cell carcinoma was correlated with poor local control, compared with primary lung tumor (SHR, 5.002; 95\% CI, 1.028 to 24.341; $\mathrm{p}=0.046)$.

After LP, six regions $(85.7 \%, 6 / 7)$ were treated with reirradiation and/or decompressive laminectomy. One region of LP was observed that was due to poor general condition without related symptoms.

Among the two Bilsky grade 3 patients who experienced LP (initial motor function grade was 5 in both patients), one patient received palliative RT 11 months after SRS (motor function, from $4+$ to 5) followed by a laminectomy an additional 9 months after the palliative RT (motor function, from 3 to 4 ). Another patient experienced aggressive LP just 1 month after SRS. Although this patient underwent laminectomy, motor function was not recovered (motor function, from 2 to 2 ).

\section{Vertebral compression fracture}

A total of 11 regions of VCF were observed. The median time of VCF was 6.5 months (range, 2.3 to 12.6 months).

The cumulative incidences of VCF at 1 year and 2 years after SRS were $7.4 \%$ and $8.3 \%$, respectively. The 1-year 

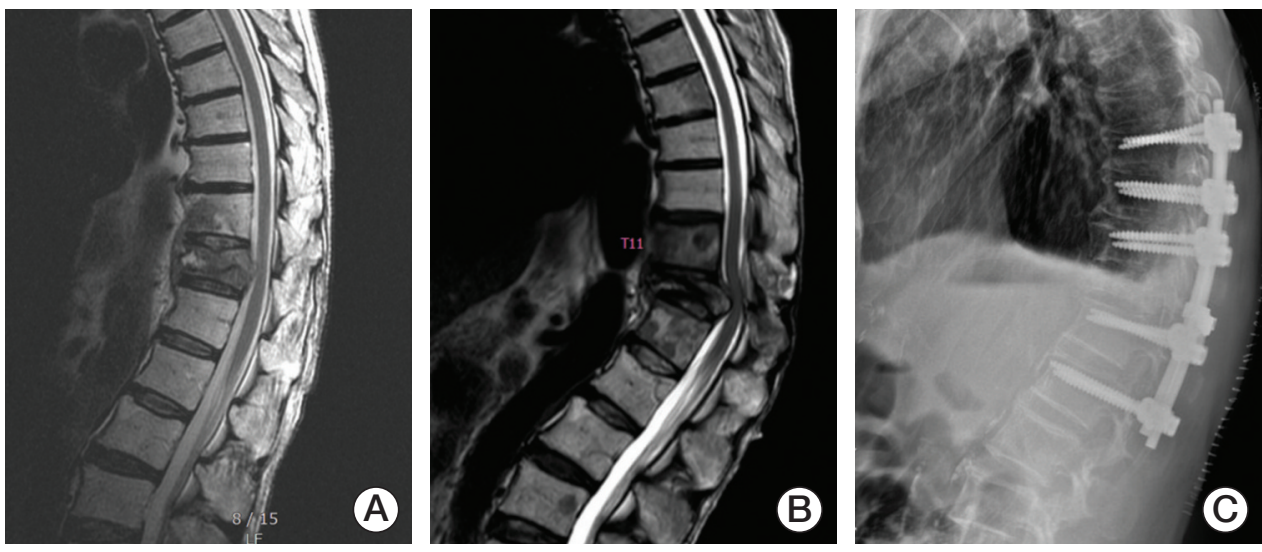

Fig. 3. An example of region (T12 spine) of progressed epidural spinal cord compression (ESCC) induced by vertebral compression fracture (VCF) after stereotactic radiosurgery (SRS) (20 Gy / 1 fraction to T11-L1 spines). (A) Before SRS (T12 spine, Bilsky grade 2). (B) Progressed VCF and ESCC at T12 spine 7 months after SRS. (C) Posterior fixation (T9- L2) after diagnosis of progressed ESCC (motor function, from 4 to $4+$ ).

Table 4. Pain response according to Bilsky grade in spinal metastasis

\begin{tabular}{lccccc} 
& \multicolumn{4}{c}{ Bilsky grade } & \multirow{2}{*}{ p-value ${ }^{a}$ Pain response } \\
\cline { 2 - 4 } & Grade 0 (n=56) & Grade 1 $(\mathrm{n}=\mathbf{3 2})$ & Grade 2 (n=15) & Grade 3 (n=8) & \\
CR & $25(44.6)$ & $6(18.8)$ & $2(13.3)$ & $1(12.5)$ & 0.008 \\
PR & $19(33.9)$ & $19(59.4)$ & $6(40.0)$ & $4(50.0)$ & \\
SD & $8(14.3)$ & $5(15.6)$ & $5(33.3)$ & $1(12.5)$ & \\
PD & $3(5.4)$ & $1(3.1)$ & $2(13.3)$ & 0 & \\
Not checkable & $1(1.8)$ & $1(3.1)$ & 0 & $2(25.0)$ & \\
\hline
\end{tabular}

Values are presented as number (\%). CR, complete response; PR, partial response; SD, stable disease; PD, progressive disease. a)Pearson's chi-squared test.

cumulative incidences of VCF for Bilsky grades $0,1,2$, and 3 were $6.6 \%, 5.2 \%, 17.1 \%$, and $12.1 \%$, respectively, and the 2-year cumulative incidences of VCF for Bilsky grades 0,1 , 2, and 3 were $7.5 \%, 5.9 \%, 19.0 \%$, and $13.6 \%$, respectively (Fig. 2). On univariate competing-risk regression, Bilsky grade was not correlated with VCF (compared with Bilsky grade 0; SHR for Bilsky grade 1, 0.781; 95\% CI, 0.154 to 3.962; $\mathrm{p}=0.766$; SHR for Bilsky grade 2, 2.733; 95\% CI, 0.635 to 11.767; $\mathrm{p}=0.177$; SHR for Bilsky grade 3, 1.890; 95\% CI, 0.224 to 15.934; $\mathrm{p}=0.559$ ) (Table 3).

According to SINS classification, cumulative incidences of VCF in the stability, intermediate stability, and instability groups at 1 year after SRS were $8.4 \%, 8.1 \%$, and $26.5 \%$, respectively. Although there was a trend of positive correlation between SINS classification and VCF risk, the number of patients in the instability group was only 4 , which may have contributed to why SINS classification did not show a statistically significant difference in VCF in the univariate com- peting risk regression.

In the univariate regressions, only the primary tumor site of the breast showed statistical significance as a favorable prognostic factor in the progression of VCF $(p<0.001)$. On multivariate competing-risk regression analysis, Bilsky grade 2 showed a significant correlation with VCF (compared with Bilsky grade 0; SHR for grade 1, 0.753; 95\% CI, 0.150 to 3.780; $\mathrm{p}=0.730$; SHR for grade 2, 5.368; 95\% CI, 1.129 to 25.530; $\mathrm{p}=0.035$; SHR for grade 3, 2.215; $95 \% \mathrm{CI}, 0.269$ to 18.248 ; $\mathrm{p}=0.460$ ).

Among the 11 regions of $\mathrm{VCF}$, four regions were treated with surgery (posterior fixation with/without decompression or corpectomy), three regions were stable without neurologic symptoms and were closely observed, three regions were not treated because of poor prognosis or patient refusal, and one region received RT because of combined LP. Among the four patients who underwent surgery, three patients showed no motor weakness (grade 5), while one experienced 
slightly improved motor function after surgery (from grade 4 to $4+$ ).

Newly diagnosed or progressed ESCC induced by VCF was observed in three $(2.0 \%, 3 / 149)$ regions, which had been Bilsky grades 0, 2, and 3. For these regions, one patient (Bilsky grade 2) underwent posterior fixation, and motor function was slightly improved (from grade 4 to $4+$ ) (Fig. 3). The other two patients did not undergo further treatment because of poor general condition and patient refusal, although they experienced motor weakness.

\section{Side effects}

Pain flare was observed in $17.1 \%$ of treatment cases (22/129) (from 2 hours after SRS to 3 days after SRS). An acute side effect was observed in 10 of 129 cases (7.8\%). Dysphasia due to RT-induced esophagitis was observed in eight of 129 cases $(6.2 \%)$. Two patients of 129 experienced grade 2 neural symptoms $(1.6 \%)$; one of them experienced a tingling sensation in both upper arms, and the other experienced radiating upper back pain with a tingling sensation as well. Side effects of grades $\geq 3$, including RT-induced neurotoxicity, were not observed.

\section{Symptom responses}

Pain at the metastatic site was observed in $86.0 \%$ of cases $(111 / 129)$. Among these cases, the complete response rate was $30.6 \%$ (34/111), the partial response rate was $43.2 \%$ (48/111), the stable disease rate was $17.1 \%$ (19/111), and the pain progression rate was $5.4 \%(6 / 111)$ (Table 4$)$. Although the pain response after SRS in patients with ESCC was lower than that in patients without ESCC, complete or partial response rates were $>50 \%$. Complete or partial response rates for Bilsky grades 0, 1, 2, and 3 were 78.5\%, 78.2\%, 53.3\%, and $62.5 \%$, respectively ( $\mathrm{p}=0.008$, Pearson's chi-squared test).

Neural defect with decreased motor function before SRS was observed in three cases. Among these, two were Bilsky grade 3 (motor function, grades 4 and 1) and one case was Bilsky grade 2 (motor function, grade 3 ). These patients did not undergo surgery because of patient refusal, short life expectancy, and/or accompanying disease. In the case with Bilsky grade 2, motor function was improved after SRS (from grade 3 to 4 ). For the cases with Bilsky grade 3, motor function level remained the same after SRS.

\section{Discussion}

Our study showed that Bilsky grade 2 ESCC did not increase the risk of LP after spinal SRS, while there was an increased, but moderate, VCF rate. There was no LP in the Bilsky grade 2 group, and among nine patients in the Bilsky grade 3 group, seven did not experience LP. Pain response rates were $53.3 \%$ and $62.5 \%$ in the grades 2 and 3 groups, respectively. During the follow-up period, no patient experienced a toxicity grade $\geq 3$. Considering these findings, SRS might be an alternative option to open surgery for patients with ESCC who do not satisfy the criteria for emergent surgical treatment.

Spinal metastasis with ESCC has been considered a contraindication for SRS because there is not sufficient space between the spinal cord and tumor [13]. The mechanism of neuropathy is considered to originate from vascular change, and a high radiation dose may provoke vascular damage and ischemia [14]. However, one study reported that as long as there is compliance with spinal cord dose constraints, neurologic damage will not be significantly higher than that in cases without ESCC [7].

In our study, prioritization of dose constraints did not decrease local control rates in the Bilsky grade 2 group. However, although most regions (7 out of 9) in the Bilsky grade 3 group showed local control during the follow-up period, the cumulative incidence of LP for Bilsky grade 3 was higher than that of grade 0 , and neurologic deficits before SRS were not improved, indicating that SRS alone might not be sufficient for patients in the grade 3 group. According to the neurologic, oncologic, mechanical, and systemic (NOMS) decision framework [15], open surgery followed by RT (SRS or conventional RT) should be considered a priority for Bilsky grade 3 patients, as long as they are in an operable condition.

There have been no published papers directly comparing surgery and SRS for spinal metastasis with ESCC [16]. The latest studies have shown that both surgery plus RT and SRS have high local control rates of approximately 80\%-90\% in ESCC patients $[17,18]$. However, SRS does not provide prompt decompression, and therefore recovery of neurological function might be less likely than is the case with surgery [19]. Surgery has a higher perioperative complication rate, while SRS is correlated with late toxicities, including VCF $[20,21]$. Therefore, SRS can be performed in patients with no/mild neurologic symptoms, who have inoperable diseases, or who are at high risk of surgical adverse events. Surgery should be considered for patients with definite neurologic symptoms, mechanical pain, or vertebral instability [22].

There were no LP events for patients with solitary or osteoblastic bone metastasis. Patients with solitary bone metasta- 
sis would have less tumor burden than those with more than one metastasis and may have undergone active treatment with a near-curative intent. These might be the reasons for higher local control rates in these patients compared to others.

There were no significant differences in primary tumors between the blastic and lytic bone quality groups. It is controversial to determine the aggressiveness of bone metastasis based on whether it is osteolytic or osteoblastic [23]. According to MD Anderson cancer response criteria for bone metastasis, sclerotic change of lytic lesions or osteoblastic flare can be considered treatment responses [24]. Since most of the patients in this study received systemic therapies, some of the osteoblastic regions might have been related to sclerotic changes, which are partial treatment responses. However, the number of patients with osteoblastic metastasis was too small to draw general conclusions.

In terms of safety, VCF after SRS appeared to cause more harm than RT-induced neurologic side effects. Not only LP of metastatic disease, but also VCF can provoke ESCC $[25,26]$. In our study, the rate of VCF-induced ESCC was $6.7 \%$ (2/30) for Bilsky grades 2-3.

SRS for spinal metastasis with ESCC may have complex effects on VCF risk. First, ESCC could mean an invasion of the metastatic tumor to the central canal, and the resulting structural instability might increase VCF risk [27]. Second, the high dose in SRS may provoke VCF [6]. And lastly, LP may generate a pathologic VCF [2]. In our study, spinal metastasis with ESCC was significantly associated with a high VCF rate after SRS, as evidenced by a 1-year cumulative incidence of $12.1 \%-17.1 \%$.

Cunha et al. [6] demonstrated that a single-dose SRS of $\geq 20$ Gy increases the VCF rate. In our study, no patient with ESCC was treated with $>20 \mathrm{~Gy}$. The instability of ESCC might lower the threshold of the RT dose for VCF to $<20 \mathrm{~Gy}$. Previous studies have reported that the VCF rate after SRS ranges from $5.7 \%$ to $41 \%$ (mean 12.3\%) [28]. Although this suggests that VCF risk in our study was moderate, considering the high rate of local control and possible instability of ESCC, reducing the RT dose for patients with ESCC might decrease SRS-induced VCF [29]. Further studies are necessary to determine an optimal RT dose for spinal metastasis with ESCC.

This study has some limitations. The nature of the study is retrospective, and the sample size was relatively small. A retrospective review about side effects may lead to underestimation of the rate of side effects. Furthermore, the performance status of the patients was poor, resulting in short follow-up periods due to death or hospital transfer to hospice care. Despite the small sample size of this study, the homogeneity of the sample-characterized by no previous spine surgery and use of single-fraction SRS only-provides accurate insight about SRS for treating spinal metastasis with ESCC.

In conclusion, after SRS for spinal cord metastasis, the presence of Bilsky grade 2 ESCC did not increase the LP rate and was associated with a moderate VCF rate. Thus, spinal SRS could be a safe and feasible treatment for spinal metastasis with Bilsky grade 2 ESCC. Since Bilsky grade 3 showed a high LP rate, SRS alone without surgery might not be appropriate for spinal metastasis with Bilsky grade 3 ESCC.

\section{Conflicts of Interest}

Conflict of interest relevant to this article was not reported.

\section{Acknowledgments}

This study was supported by the 2017 Young Medical Science Researcher Grants from Ewha Womans University College of Medicine.

\section{References}

1. Byrne TN. Spinal cord compression from epidural metastases. N Engl J Med. 1992;327:614-9.

2. Patchell RA, Tibbs PA, Regine WF, Payne R, Saris S, Kryscio $\mathrm{RJ}$, et al. Direct decompressive surgical resection in the treatment of spinal cord compression caused by metastatic cancer: a randomised trial. Lancet. 2005;366:643-8.

3. Pan H, Simpson DR, Mell LK, Mundt AJ, Lawson JD. A survey of stereotactic body radiotherapy use in the United States. Cancer. 2011;117:4566-72.

4. Yu JI, Park HC, Ahn YC, Chung Y, Koom WS, Song SY. Vari- ation in practice patterns of Korean radiation oncologists for spine metastasis between 2009 and 2014. Cancer Res Treat. 2016;48:1102-9.

5. Yamada Y, Katsoulakis E, Laufer I, Lovelock M, Barzilai O, McLaughlin LA, et al. The impact of histology and delivered dose on local control of spinal metastases treated with stereotactic radiosurgery. Neurosurg Focus. 2017;42:E6.

6. Cunha MV, Al-Omair A, Atenafu EG, Masucci GL, Letourneau D, Korol R, et al. Vertebral compression fracture (VCF) after spine stereotactic body radiation therapy (SBRT): analysis 
of predictive factors. Int J Radiat Oncol Biol Phys. 2012;84: e343-9.

7. Kirkpatrick JP, van der Kogel AJ, Schultheiss TE. Radiation dose-volume effects in the spinal cord. Int J Radiat Oncol Biol Phys. 2010;76(3 Suppl):S42-9.

8. Cox BW, Spratt DE, Lovelock M, Bilsky MH, Lis E, Ryu S, et al. International Spine Radiosurgery Consortium consensus guidelines for target volume definition in spinal stereotactic radiosurgery. Int J Radiat Oncol Biol Phys. 2012;83:e597-605.

9. Bilsky MH, Laufer I, Fourney DR, Groff M, Schmidt MH, Varga PP, et al. Reliability analysis of the epidural spinal cord compression scale. J Neurosurg Spine. 2010;13:324-8.

10. Fisher CG, DiPaola CP, Ryken TC, Bilsky MH, Shaffrey CI, Berven $\mathrm{SH}$, et al. A novel classification system for spinal instability in neoplastic disease: an evidence-based approach and expert consensus from the Spine Oncology Study Group. Spine (Phila Pa 1976). 2010;35:E1221-9.

11. Cox JD, Stetz J, Pajak TF. Toxicity criteria of the Radiation Therapy Oncology Group (RTOG) and the European Organization for Research and Treatment of Cancer (EORTC). Int J Radiat Oncol Biol Phys. 1995;31:1341-6.

12. Fine JP, Gray RJ. A proportional hazards model for the subdistribution of a competing risk. J Am Stat Assoc. 1999;94:496509.

13. Wang XS, Rhines LD, Shiu AS, Yang JN, Selek U, Gning I, et al. Stereotactic body radiation therapy for management of spinal metastases in patients without spinal cord compression: a phase 1-2 trial. Lancet Oncol. 2012;13:395-402.

14. St Clair WH, Arnold SM, Sloan AE, Regine WF. Spinal cord and peripheral nerve injury: current management and investigations. Semin Radiat Oncol. 2003;13:322-32.

15. Laufer I, Rubin DG, Lis E, Cox BW, Stubblefield MD, Yamada $\mathrm{Y}$, et al. The NOMS framework: approach to the treatment of spinal metastatic tumors. Oncologist. 2013;18:744-51.

16. Suppli MH, Af Rosenschold PM, Pappot H, Dahl B, Morgen SS, Vogelius IR, et al. Stereotactic radiosurgery versus decompressive surgery followed by postoperative radiotherapy for metastatic spinal cord compression (STEREOCORD): Study protocol of a randomized non-inferiority trial. J Radiosurg SBRT. 2016;4:S1-9.

17. Pessina F, Navarria P, Riva M, Franceschini D, Nibali MC, Fornari M, et al. Long-term follow-up of patients with metastatic epidural spinal cord compression from breast cancer treated with surgery followed by radiotherapy. World Neurosurg. 2018;110:e281-6.

18. Ghia AJ, Guha-Thakurta N, Hess K, Yang JN, Settle SH, Sharpe HJ, et al. Phase 1 study of spinal cord constraint relax- ation with single session spine stereotactic radiosurgery in the primary management of patients with inoperable, previously unirradiated metastatic epidural spinal cord compression. Int J Radiat Oncol Biol Phys. 2018;102:1481-8.

19. Vellayappan BA, Kumar N, Chang EL, Sahgal A, Sloan AE, Lo SS. Novel multidisciplinary approaches in the management of metastatic epidural spinal cord compression. Future Oncol. 2018;14:1665-8.

20. Park SJ, Lee CS, Chung SS. Surgical results of metastatic spinal cord compression (MSCC) from non-small cell lung cancer (NSCLC): analysis of functional outcome, survival time, and complication. Spine J. 2016;16:322-8.

21. Husain ZA, Sahgal A, De Salles A, Funaro M, Glover J, Hayashi M, et al. Stereotactic body radiotherapy for de novo spinal metastases: systematic review. J Neurosurg Spine. 2017;27:295-302.

22. Spratt DE, Beeler WH, de Moraes FY, Rhines LD, Gemmete JJ, Chaudhary N, et al. An integrated multidisciplinary algorithm for the management of spinal metastases: an International Spine Oncology Consortium report. Lancet Oncol. 2017;18: e720-30.

23. Roodman GD. Mechanisms of bone metastasis. N Engl J Med. 2004;350:1655-64.

24. Costelloe CM, Chuang HH, Madewell JE, Ueno NT. Cancer response criteria and bone metastases: RECIST 1.1, MDA and PERCIST. J Cancer. 2010;1:80-92.

25. Virk MS, Han JE, Reiner AS, McLaughlin LA, Sciubba DM, Lis E, et al. Frequency of symptomatic vertebral body compression fractures requiring intervention following single-fraction stereotactic radiosurgery for spinal metastases. Neurosurg Focus. 2017;42:E8.

26. Al-Omair A, Smith R, Kiehl TR, Lao L, Yu E, Massicotte EM, et al. Radiation-induced vertebral compression fracture following spine stereotactic radiosurgery: clinicopathological correlation. J Neurosurg Spine. 2013;18:430-5.

27. Sahgal A, Atenafu EG, Chao S, Al-Omair A, Boehling N, Balagamwala $\mathrm{EH}$, et al. Vertebral compression fracture after spine stereotactic body radiotherapy: a multi-institutional analysis with a focus on radiation dose and the spinal instability neoplastic score. J Clin Oncol. 2013;31:3426-31.

28. Evans JD, Brown PD, Olivier KR. Stereotactic spinal radiosurgery and delayed vertebral fracture risk. Adv Radiat Oncol. 2019;4:20-5.

29. Boyce-Fappiano D, Elibe E, Schultz L, Ryu S, Siddiqui MS, Chetty I, et al. Analysis of the factors contributing to vertebral compression fractures after spine stereotactic radiosurgery. Int J Radiat Oncol Biol Phys. 2017;97:236-45. 